\title{
Trastuzumab/Nab-paclitaxel Regimen
}

National Cancer Institute

\section{Source}

National Cancer Institute. Trastuzumab/Nab-paclitaxel Regimen. NCI Thesaurus. Code C156372.

A chemotherapy regimen consisting of trastuzumab and nab-paclitaxel, an albuminbound formulation of paclitaxel, that may be used for the treatment of HER-2 (EGFR2; ErbB2)-positive breast cancer. 